\title{
Development of surface plasmon resonance sensor for determining zinc ion using novel active nanolayers as probe
}

\begin{abstract}
In this study, novel active nanolayers in combination with surface plasmon resonance (SPR) system for zinc ion (Zn2+) detection has been developed. The gold surface used for the SPR system was modified with the novel developed active nanolayers, i.e. chitosan and chitosantetrabutyl thiuram disulfide (chitosan-TBTDS). Both chitosan and chitosan-TBTDS active layers were fabricated on the gold surface by spin coating technique. The system was used to monitor SPR signal for $\mathrm{Zn} 2+$ in aqueous media with and without sensitivity enhancement by TBTDS. For both active nanolayers, the shift of resonance angle is directly proportional to the concentration of $\mathrm{Zn} 2+$ in aqueous media. The higher shift of resonance angle was obtained for chitosan-TBTDS active nanolayer due to a specific binding of TBTDS with $\mathrm{Zn} 2+$. The chitosan-TBTDS active nanolayer enhanced the sensitivity of detection down to $0.1 \mathrm{mg} / \mathrm{l}$ and also induced a selective detection towards $\mathrm{Zn} 2+$.
\end{abstract}

Keyword: Surface plasmon resonance; Zinc ion; Novel active nanolayer; Chitosan; Tetrabutyl thiuram disulfide 